\title{
Entrepreneurial Intentions of the Graduating Students in Oman: A Case Study - Sohar university
}

\author{
Riham Al-Jahwari, Firdouse Rahman Khan, Rofida Al-Shibli \& Najoom Al-Dahoori \\ Faculty of Business, Sohar University, Sultanate of Oman \\ Email: ${ }^{1}$ Rihamnasser.aljahwari@gmail.com,2firdouse@yahoo.co.uk, ${ }^{3}$ rofidaalshibli@gmail.com \& \\ Najoom9976@gmail.com
}

Article History: Received on $21^{\text {st }}$ Mar. 2020, Revised on $17^{\text {th }}$ Apr. 2020, Published on $31^{\text {st }}$ July 2020

Abstract
Purpose of the study: The objective of the study is to critically do a review of literature on the factors
impacting the entrepreneurial intention of the graduating students and to critically analyze the impact of such
factors on the entrepreneurial intention of the graduating students.
Design/Methodology: 269 undergraduate students were selected on a random sampling basis from Sohar
University and were considered to be the sample for the study. An online questionnaire was shared through
digital media for survey purposes which were filled by students. The statistical analysis was carried out using
excel and SPSS.

Findings: The findings of the study reveal that the Entrepreneurial intention is influenced by Graduate Potentials, Creativity, and Education of the students and not by Government support. The undergraduate students are developing creative skills and bringing out their entrepreneurial potentials and improvising their practical knowledge through their education.

Implications: It was suggested that the graduating students need proper guidance to fulfill their entrepreneurial intention which is in the hands of teachers. Students should be exposed to the development of various skills - creativity, communication, presentation, marketing, funds management, etc. and they should be invited to a platform where competitions of all innovative kinds should be conducted regularly and the participants should be guided with assistance to improve their ideas.

Originality: This research work is one of its first kind as no study was conducted before focusing on the entrepreneurial intentions of the students in Oman.

Keywords: Entrepreneurial Intention, Entrepreneurial Potentials, Creativity, Government support, Education.

\section{Introduction}

Entrepreneurship is the process of creating a new business that has not previously existed or developing a business with the opportunities available to develop and advance innovatively (Santarelli \& Vivarelli, 2007). The fact that entrepreneurship fulfils the dream of the owner of an entrepreneurial idea to apply it on the field, and thus constitutes a new source of jobs to the labour market and source of income to improve the living condition. Also, it aims to develop and support a specific project that would raise the level of economic and technological performance of the country. Entrepreneurship is recognized as an important source of job growth and economic development for a country and is not limited to megaprojects but rather small projects that include small companies with limited responsibility, in-country value projects, and petty commercial businesses (Audretsch \& Thurik, 2001).

Entrepreneurs are considered as crucial growth agents of a nation to achieve economic, social, technological and organizational development and they serve as catalysts for economic development due to the wide array of economic benefits provided by them in terms of introducing innovative products, services, job creation, facilitating technology transfer from lab to land, increasing competitiveness and enabling social empowerment (Ács et al., 2014; Bosma et al., 2018; Fayolle et al., 2016). Therefore, policymakers across the globe are emphasizing on numerous entrepreneurship support measures (Gurol \& Atsan, 2006). Entrepreneurs are considered as the backbone of the industries as they come up with innovative business ideas which ultimately contribute to the social and economic growth (Khan, 2014).

In the Sultanate of Oman, thousands of students graduate from universities/colleges every year, but only a few of them intend to start their own businesses (Matlay et al., 2015). This might be due to the lack of awareness and interest in entrepreneurship, especially those who are graduating. They also not aware of the fact that the Government is providing support to graduated youth to start an entrepreneurial venture and also there are companies providing support to help the student but with certain conditions conducting entrepreneurship competitions (Khan et al., 2005). Higher Education Institutions (HEIs) in Oman have also 
introduced entrepreneurship as a part of their curriculum to motivate the students by making it a compulsory subject as per the directive of the Ministry of Higher Education, Oman (Bindah \& Magd, 2016). Thus, the increase in the business knowledge of graduates and business risk understanding influences them towards entrepreneurship (Ibrahim et al., 2017). Though such entrepreneurial potentials (EP) are enhanced the rate of entrepreneurial ventures is very low. An individual with EP is one who bears risks, works under uncertainty, combines and manages capital, innovates on all fronts on regular basis, and is motivated by business results (Krueger \& Brazeal, 1994, Sahay \& Rai, 2004).

Thus, it has become necessary to find out the factors impeding the entrepreneurial intentions of the graduating students towards starting businesses and their knowledge and creativity towards entrepreneurship.

Therefore, the following research questions arise:

\section{Research Questions}

1. What are the factors impacting the Entrepreneurial Intention of graduating students?

2. How do the factors impact the Entrepreneurial intention of graduating students?

Based on the above research questions, it is defined that the objective of the study is to critically analyze the review of literature on the factors impacting the entrepreneurial intention of the graduating students and to critically analyze the impact of such factors on the entrepreneurial intention of the graduating students.

\section{Literature Review \\ Entrepreneurial Potentials}

Circumstances and traits have driven and continue to drive 'real-life' entrepreneurs in students who have entrepreneurial potential (Galloway et al., 2006). Entrepreneurial potential (EP) is a measure of individual capability and desire to become an entrepreneur and is recognized as an important precursor to entrepreneurial events or activities (Krueger \& Brazeal, 1994). Entrepreneurial potential on which the two groups differed was their predisposition to risk-taking. Females were less disposed to taking risks and it is not true that the female student in middle-east countries may be less averse to entrepreneurial activities (Zeffane, 2013). The classification of idea factors will identify the go or no-go decision for a new entrepreneur and the selection process for a business based on their personality traits and preferences (Wong et al., 2005). The personality traits of students indirectly influence their intentions to start a new business through their attitudes (Lüthje and Franke, 2003). Young students are ambitious and energetic, and love to venture into entrepreneurial businesses by providing what is new, useful - innovation (Haddad et al., 2019). Undergraduate students have a high level of potential to become entrepreneurs and they are considered as the source of potential entrepreneurs (Postigo et al., 2003). As Engineering students reported to have many enterprise skills, many of them aim to start firms as they understand the enterprise skills which are relevant to employment and personal development (Galloway et al., 2006).

\section{Government Support}

The Government has an important role to play in changing the attitude of graduates towards entrepreneurship and setting effective entrepreneurship strategies (Ibrahim et al., 2017). In an attempt to diversify the economy and stimulate private enterprise development, government agencies and private institutions are participating in different entrepreneurship development activities (Khan \& Al Moharby, 2007). Considering the importance of entrepreneurship in the growth of countries, the Government of Oman is trying entrepreneurship education towards people who have an entrepreneurial mind (Yarahmadi \& Magd, 2016). Students are not aware of whether Government support to start a business is available in Oman (Varghese \& Hassan, 2012). Indeed, Government support is extended in Oman through the ministries across a range of government bodies from labor to technology and education to industrial development (Ennis, 2015). Big merchant families also back Government support by playing key roles in Oman's Chamber of Commerce (Valeri, 2013).

\section{Creativity}

Creativity adds excellence and enhances the capability of students toward creative thinking and creativity is seen as a behavior that generates useful ideas for the development (Mathisen et al., 2012). Entrepreneurs determine their future themselves and go into the battlefield of work and planning towards job creation (Hamdan, 2019). The central role of creativity and innovation in entrepreneurship is in the generation of new ideas which has commercial and social value (Matriano \& Suguku, 2015). Crowdfunding has a role in boosting the entrepreneurial creativity of university students and the business incubators at universities promote creative innovation, by providing support for their transformation into projects through crowdfunding (El Talla et al., 2018). The educational system, in Oman, has been accused of not encouraging 
innovation and creativity, but rather promoting memorizing and imitating (Al-Shanfari, 2012). The culture of creativity depends on many factors such as structural characteristics, personality traits, and management practices (Y1lmaz, 2010). The perception of the relationship between the person and the environment and creativity is conditioned by the personality traits and the different perceptions of the surrounding world (Durrah, et al., 2018; Sokol et al., 2015).

\section{Education}

Education and new venture creation are positively and significantly correlated (Hunjra et al., 2011). There is a relationship between the individuals' educational level and their probability of becoming entrepreneurs (Cowling and Taylor, 2001; Delmar and Davidsson, 2000). The role of higher education is essential to develop the entrepreneurial potential and the academic environment may be useful to foster a more favorable atmosphere for innovation and entrepreneurship (Marques \& Moreira, 2013). The long-term view of the entrepreneurial potential of graduate entrepreneurship is only feasible as new graduates lack the resources, skills, and experience necessary for sustainability and growth of ventures (Galloway \& Brown, 2002).

\section{Hypotheses}

Based on the above review of literature, the independent variables considered are Entrepreneurial Potential, Government support, Creativity, and Education. Thus, the following hypotheses were derived:

1. Entrepreneurial Potential influences the entrepreneurial intention of the students

2. Government support impacts the entrepreneurial intention of the students

3. Creativity influences the entrepreneurial intention of the students

4. Education impact the entrepreneurial intention of the students.

\section{Research Methodology}

The research work was done to study the entrepreneurial intentions of graduating students at Sohar University. 269 undergraduate students were selected on a random sampling basis from Sohar University and were considered to be the sample for the study. An online questionnaire was shared through digital media for survey purposes which were filled by students. The statistical analysis was carried out using excel and SPSS.

\section{Findings}

Table.1 Reliability Analysis

\begin{tabular}{|c|c|c|}
\hline & $\mathrm{N}$ & $\%$ \\
\hline Valid Cases & 269 & 100.0 \\
\hline Excluded & 0 & 0.0 \\
\hline
\end{tabular}

\begin{tabular}{|c|c|}
\hline Cronbach's Alpha & No. of Items \\
\hline .953 & 35 \\
\hline
\end{tabular}

The reliability analysis shows that the Cronbach Alpha score $=0.953>0.7$

Table 2 Demographic Details

\begin{tabular}{|l|l|c|c|}
\hline & & Frequency & \% \\
\hline \multirow{3}{*}{ Gender } & Male & 215 & $79.9 \%$ \\
\cline { 2 - 4 } & Female & 54 & $20.1 \%$ \\
\hline \multirow{5}{*}{ Age } & $20-<25$ & 196 & $72.9 \%$ \\
\cline { 2 - 4 } & $25-<30$ & 44 & $16.4 \%$ \\
\cline { 2 - 4 } & $30-<35$ & 20 & $7.4 \%$ \\
\cline { 2 - 4 } & 30 above & 9 & $3.3 \%$ \\
\hline \multirow{3}{*}{ Working status } & Working & 74 & $27.5 \%$ \\
\cline { 2 - 4 } & Not working & 195 & $72.5 \%$ \\
\hline \multirow{2}{*}{ Want to establish new } & Yes & 194 & $72.1 \%$ \\
\cline { 2 - 4 } Willing to give up jobs & No & 75 & $27.9 \%$ \\
\cline { 2 - 4 } & Yes & 102 & $62.1 \%$ \\
\cline { 2 - 4 } & No & & $37.9 \%$ \\
\hline
\end{tabular}




\begin{tabular}{|c|c|c|c|}
\hline \multirow{2}{*}{ Entrepreneurs in family } & Yes & 179 & $66.5 \%$ \\
\hline & No & 90 & $33.5 \%$ \\
\hline \multirow{2}{*}{$\begin{array}{l}\text { Have the confidence to } \\
\text { start a business }\end{array}$} & Yes & 173 & $64.3 \%$ \\
\hline & No & 96 & $35.7 \%$ \\
\hline \multirow{4}{*}{$\begin{array}{l}\text { The main reason for } \\
\text { starting their own } \\
\text { business }\end{array}$} & $\begin{array}{l}\text { The success story of } \\
\text { entrepreneurs }\end{array}$ & 50 & $18.6 \%$ \\
\hline & Trg \& dev. opportunities & 70 & $26.0 \%$ \\
\hline & Financial support & 53 & $19.7 \%$ \\
\hline & Family support & 52 & $19.3 \%$ \\
\hline
\end{tabular}

Source: Questionnaire

Table.3 Entrepreneurial Potentials

\begin{tabular}{|c|c|c|c|c|c|c|c|c|}
\hline Statements & SA & $\mathbf{A}$ & $\mathbf{N}$ & D & SD & $\begin{array}{c}\text { K-S } \\
\text { value }\end{array}$ & $\chi^{2}$ & $\begin{array}{c}\text { p- } \\
\text { value }\end{array}$ \\
\hline $\begin{array}{l}\text { The graduate has skills in } \\
\text { building relationships } \\
\text { with others }\end{array}$ & $\begin{array}{c}37 \\
13.8 \%\end{array}$ & $\begin{array}{c}94 \\
34.9 \%\end{array}$ & $\begin{array}{c}94 \\
34.9 \%\end{array}$ & $\begin{array}{l}18 \\
6.7 \%\end{array}$ & $\begin{array}{c}26 \\
9.7 \%\end{array}$ & .207 & \multirow{7}{*}{140.216} & \multirow{7}{*}{.000} \\
\hline $\begin{array}{l}\text { The graduate has the } \\
\text { skills to take } \\
\text { responsibility }\end{array}$ & $\begin{array}{c}52 \\
19.3 \%\end{array}$ & $\begin{array}{c}105 \\
39.0 \%\end{array}$ & $\begin{array}{c}63 \\
23.4 \%\end{array}$ & $\begin{array}{c}30 \\
11.2 \%\end{array}$ & $\begin{array}{c}19 \\
7.1 \%\end{array}$ & .246 & & \\
\hline $\begin{array}{l}\text { The necessary knowledge } \\
\text { was acquired to meet the } \\
\text { labour market needs }\end{array}$ & $\begin{array}{c}41 \\
15.2 \%\end{array}$ & $\begin{array}{c}86 \\
32.0 \%\end{array}$ & $\begin{array}{c}87 \\
32.3 \%\end{array}$ & $\begin{array}{c}38 \\
14.1 \%\end{array}$ & $\begin{array}{c}17 \\
6.3 \%\end{array}$ & .194 & & \\
\hline $\begin{array}{l}\text { The graduate has effective } \\
\text { communication skills }\end{array}$ & $\begin{array}{c}49 \\
18.2 \% \\
\end{array}$ & $\begin{array}{c}104 \\
38.7 \% \\
\end{array}$ & $\begin{array}{c}77 \\
28.6 \% \\
\end{array}$ & $\begin{array}{c}27 \\
10.0 \% \\
\end{array}$ & $\begin{array}{c}12 \\
4.5 \% \\
\end{array}$ & .232 & & \\
\hline $\begin{array}{l}\text { I prefer to overcome } \\
\text { challenges than to create } \\
\text { new opportunities }\end{array}$ & $\begin{array}{c}50 \\
18.6 \%\end{array}$ & $\begin{array}{c}97 \\
36.1 \%\end{array}$ & $\begin{array}{c}78 \\
29.0 \%\end{array}$ & $\begin{array}{c}26 \\
9.7 \%\end{array}$ & $\begin{array}{c}18 \\
6.7 \%\end{array}$ & .220 & & \\
\hline $\begin{array}{l}\text { The graduate can } \\
\text { accomplish several tasks }\end{array}$ & $\begin{array}{c}65 \\
24.2 \% \\
\end{array}$ & $\begin{array}{c}99 \\
36.8 \% \\
\end{array}$ & $\begin{array}{c}68 \\
25.3 \% \\
\end{array}$ & $\begin{array}{c}19 \\
7.1 \% \\
\end{array}$ & $\begin{array}{c}18 \\
6.7 \% \\
\end{array}$ & .233 & & \\
\hline $\begin{array}{l}\text { The graduates have the } \\
\text { confidence to start a } \\
\text { business }\end{array}$ & $\begin{array}{c}60 \\
22.3 \%\end{array}$ & $\begin{array}{c}112 \\
41.6 \%\end{array}$ & $\begin{array}{c}57 \\
21.2 \%\end{array}$ & $\begin{array}{c}22 \\
8.2 \%\end{array}$ & $\begin{array}{c}18 \\
6.7 \%\end{array}$ & .264 & & \\
\hline
\end{tabular}

Null Hypotheses.1: There is no relationship between the choice of the respondents and the Graduates Potentials.

From table No.3, it is observed that the p-value is less than 0.05 which means that the null hypothesis is rejected. This means that there is a clear relationship between the choices of the respondents and the Graduates Potentials. Comparing the K-S values that were obtained from the Kolmogorov-Smirnov test, it can be observed that 'The graduates have the confidence to start a business' ranked first (.264), followed by, 'The graduate has the skills to take responsibility' (.246), and 'The graduate can accomplish several tasks' $(.233)$.

Table.4 Government Support

\begin{tabular}{|c|c|c|c|c|c|c|c|c|}
\hline Statements & SA & $\mathbf{A}$ & $\mathbf{N}$ & D & SD & $\begin{array}{c}\text { K-S } \\
\text { Value }\end{array}$ & $\chi^{2}$ & $\begin{array}{c}\text { p- } \\
\text { value }\end{array}$ \\
\hline
\end{tabular}




\begin{tabular}{|c|c|c|c|c|c|c|c|c|}
\hline $\begin{array}{l}\text { Help from the Government } \\
\text { encourages starting a business }\end{array}$ & $\begin{array}{c}70 \\
26.0 \%\end{array}$ & $\begin{array}{c}84 \\
31.2 \%\end{array}$ & $\begin{array}{c}72 \\
26.8 \%\end{array}$ & $\begin{array}{c}26 \\
9.7 \%\end{array}$ & $\begin{array}{c}17 \\
6.3 \%\end{array}$ & .205 & \multirow{7}{*}{241.333} & \multirow{7}{*}{.000} \\
\hline $\begin{array}{l}\text { Project control by the } \\
\text { Government since its beginning } \\
\text { makes the project successful }\end{array}$ & $\begin{array}{c}57 \\
21.2 \%\end{array}$ & $\begin{array}{c}101 \\
37.5 \%\end{array}$ & $\begin{array}{c}76 \\
28.3 \%\end{array}$ & $\begin{array}{c}22 \\
8.2 \%\end{array}$ & $\begin{array}{c}13 \\
4.8 \%\end{array}$ & .227 & & \\
\hline $\begin{array}{l}\text { New ideas by students need } \\
\text { Government support to put into } \\
\text { use }\end{array}$ & $\begin{array}{c}88 \\
32.7 \%\end{array}$ & $\begin{array}{c}109 \\
40.5 \%\end{array}$ & $\begin{array}{c}46 \\
17.1 \%\end{array}$ & $\begin{array}{c}11 \\
4.1 \%\end{array}$ & $\begin{array}{c}15 \\
5.6 \%\end{array}$ & .267 & & \\
\hline $\begin{array}{l}\text { Emotional support from family } \\
\text { and friends makes proud }\end{array}$ & $\begin{array}{c}120 \\
44.6 \%\end{array}$ & $\begin{array}{c}76 \\
28.3 \%\end{array}$ & $\begin{array}{c}54 \\
20.1 \%\end{array}$ & $\begin{array}{c}8 \\
3.0 \%\end{array}$ & $\begin{array}{c}11 \\
4.1 \%\end{array}$ & .257 & & \\
\hline $\begin{array}{l}\text { I am sure the Government will } \\
\text { support to start a project }\end{array}$ & $\begin{array}{c}41 \\
15.2 \% \\
\end{array}$ & $\begin{array}{c}85 \\
31.6 \% \\
\end{array}$ & $\begin{array}{c}85 \\
31.6 \% \\
\end{array}$ & $\begin{array}{c}41 \\
15.2 \% \\
\end{array}$ & $\begin{array}{c}17 \\
6.3 \% \\
\end{array}$ & .193 & & \\
\hline $\begin{array}{l}\text { Government supports projects } \\
\text { to grow }\end{array}$ & $\begin{array}{c}47 \\
17.5 \% \\
\end{array}$ & $\begin{array}{c}107 \\
39.8 \% \\
\end{array}$ & $\begin{array}{c}85 \\
31.6 \% \\
\end{array}$ & $\begin{array}{c}15 \\
5.6 \% \\
\end{array}$ & $\begin{array}{c}15 \\
5.6 \% \\
\end{array}$ & .232 & & \\
\hline $\begin{array}{l}\text { The government encourages } \\
\text { graduates to become } \\
\text { entrepreneurs }\end{array}$ & $\begin{array}{c}44 \\
16.4 \%\end{array}$ & $\begin{array}{c}97 \\
36.1 \%\end{array}$ & $\begin{array}{c}85 \\
31.6 \%\end{array}$ & $\begin{array}{c}23 \\
8.6 \%\end{array}$ & $\begin{array}{c}18 \\
6.7 \%\end{array}$ & .216 & & \\
\hline
\end{tabular}

Null Hypotheses.2: There is no relationship between the choice of the respondents and the Government support.

From table No.4, it is observed that the p-value is less than 0.05 which means that the null hypothesis is rejected. This means that there is a clear relationship between the choices of the respondents and the Government support. Comparing the K-S values that were obtained from the Kolmogorov-Smirnov test, it can be observed that 'New ideas by students need Government support to put into use' ranked first (.267), followed by, 'Emotional support from family and friends makes proud' (.257), and 'Government supports projects to grow' (.232).

Table.5 Creativity

\begin{tabular}{|c|c|c|c|c|c|c|c|c|}
\hline Statements & SA & $\mathbf{A}$ & $\mathbf{N}$ & D & SD & $\begin{array}{c}\mathrm{K}-\mathrm{S} \\
\text { value }\end{array}$ & $\chi^{2}$ & $\begin{array}{c}\text { p- } \\
\text { value }\end{array}$ \\
\hline $\begin{array}{l}\text { I can do business in a new \& } \\
\text { interesting way }\end{array}$ & $\begin{array}{c}43 \\
16.0 \% \\
\end{array}$ & $\begin{array}{c}123 \\
45.7 \% \\
\end{array}$ & $\begin{array}{c}70 \\
26.0 \% \\
\end{array}$ & $\begin{array}{c}20 \\
7.4 \% \\
\end{array}$ & $\begin{array}{c}13 \\
4.8 \% \\
\end{array}$ & .270 & \multirow{7}{*}{187.963} & \multirow{7}{*}{.000} \\
\hline $\begin{array}{l}\text { The products should be } \\
\text { different and better }\end{array}$ & $\begin{array}{c}105 \\
39.0 \%\end{array}$ & $\begin{array}{c}102 \\
37.9 \%\end{array}$ & $\begin{array}{c}33 \\
12.3 \%\end{array}$ & $\begin{array}{c}15 \\
5.6 \%\end{array}$ & $\begin{array}{c}12 \\
4.5 \%\end{array}$ & .267 & & \\
\hline $\begin{array}{l}\text { Creativity is essential for a } \\
\text { new business }\end{array}$ & $\begin{array}{c}109 \\
40.5 \%\end{array}$ & $\begin{array}{c}99 \\
36.8 \%\end{array}$ & $\begin{array}{c}35 \\
13.0 \%\end{array}$ & $\begin{array}{c}17 \\
6.3 \%\end{array}$ & $\begin{array}{c}9 \\
3.3 \%\end{array}$ & .255 & & \\
\hline $\begin{array}{l}\text { I have creative talents to } \\
\text { become an entrepreneur }\end{array}$ & $\begin{array}{c}66 \\
24.5 \%\end{array}$ & $\begin{array}{c}117 \\
43.5 \%\end{array}$ & $\begin{array}{c}61 \\
22.7 \%\end{array}$ & $\begin{array}{c}14 \\
5.2 \%\end{array}$ & $\begin{array}{c}11 \\
4.1 \%\end{array}$ & .262 & & \\
\hline $\begin{array}{l}\text { My creative skills encourages } \\
\text { me to engage in } \\
\text { entrepreneurship }\end{array}$ & $\begin{array}{c}52 \\
19.3 \%\end{array}$ & $\begin{array}{c}120 \\
44.6 \%\end{array}$ & $\begin{array}{c}74 \\
27.5 \%\end{array}$ & $\begin{array}{c}15 \\
5.6 \%\end{array}$ & $\begin{array}{c}8 \\
3.0 \%\end{array}$ & .258 & & \\
\hline $\begin{array}{l}\text { I have the ability to present } \\
\text { ideas }\end{array}$ & $\begin{array}{c}65 \\
24.2 \%\end{array}$ & $\begin{array}{c}127 \\
47.2 \%\end{array}$ & $\begin{array}{c}57 \\
21.2 \%\end{array}$ & $\begin{array}{c}11 \\
4.1 \%\end{array}$ & $\begin{array}{c}9 \\
3.3 \%\end{array}$ & .278 & & \\
\hline $\begin{array}{l}\text { I can find solutions to } \\
\text { problems }\end{array}$ & $\begin{array}{c}67 \\
24.9 \% \\
\end{array}$ & $\begin{array}{c}123 \\
45.7 \% \\
\end{array}$ & $\begin{array}{c}53 \\
19.7 \% \\
\end{array}$ & $\begin{array}{c}13 \\
4.8 \% \\
\end{array}$ & $\begin{array}{c}13 \\
4.8 \% \\
\end{array}$ & .280 & & \\
\hline
\end{tabular}

Null Hypotheses.3: There is no relationship between the choice of the respondents and Creativity.

From table No.5, it is observed that the p-value is less than 0.05 which means that the null hypothesis is rejected. This means that there is a clear relationship between the choices of the respondents and Creativity. Comparing the K-S values that were obtained from the Kolmogorov-Smirnov test, it can be observed that 'I can find solutions to problems' ranked first (.280), followed by, 'I can present ideas' (.278), and 'I can do business in a new \& interesting way' (.270). 
Table.6 Education

\begin{tabular}{|c|c|c|c|c|c|c|c|c|}
\hline Statements & $\mathbf{S A}$ & $\mathbf{A}$ & $\mathbf{N}$ & D & SD & $\begin{array}{c}\text { K-S } \\
\text { value }\end{array}$ & $\chi^{2}$ & $\begin{array}{c}\text { p- } \\
\text { value }\end{array}$ \\
\hline $\begin{array}{l}\text { Entrepreneurship education gives } \\
\text { students business knowledge and a } \\
\text { better understanding }\end{array}$ & $\begin{array}{c}65 \\
24.2 \%\end{array}$ & $\begin{array}{c}111 \\
41.3 \%\end{array}$ & $\begin{array}{c}58 \\
21.6 \%\end{array}$ & $\begin{array}{c}22 \\
8.2 \%\end{array}$ & $\begin{array}{c}13 \\
4.8 \%\end{array}$ & .258 & \multirow{7}{*}{204.253} & \multirow{7}{*}{.000} \\
\hline $\begin{array}{l}\text { Working in a small business } \\
\text { makes students gain real } \\
\text { experience }\end{array}$ & $\begin{array}{c}76 \\
28.3 \%\end{array}$ & $\begin{array}{c}110 \\
40.9 \%\end{array}$ & $\begin{array}{c}60 \\
22.3 \%\end{array}$ & $\begin{array}{c}11 \\
4.1 \%\end{array}$ & $\begin{array}{c}12 \\
4.5 \%\end{array}$ & .252 & & \\
\hline $\begin{array}{l}\text { Entrepreneurship education } \\
\text { competitions inspires and } \\
\text { motivates }\end{array}$ & $\begin{array}{c}78 \\
29.0 \%\end{array}$ & $\begin{array}{c}92 \\
34.2 \%\end{array}$ & $\begin{array}{c}67 \\
24.9 \%\end{array}$ & $\begin{array}{c}19 \\
7.1 \%\end{array}$ & $\begin{array}{c}13 \\
4.8 \%\end{array}$ & .221 & & \\
\hline $\begin{array}{l}\text { Students attending entrepreneurial } \\
\text { competitions needs emotional } \\
\text { support }\end{array}$ & $\begin{array}{c}93 \\
34.6 \%\end{array}$ & $\begin{array}{c}102 \\
37.9 \%\end{array}$ & $\begin{array}{c}53 \\
19.7 \%\end{array}$ & $\begin{array}{c}11 \\
4.1 \%\end{array}$ & $\begin{array}{c}10 \\
3.7 \%\end{array}$ & .242 & & \\
\hline $\begin{array}{l}\text { Entrepreneurial activities increased } \\
\text { my leadership qualities }\end{array}$ & $\begin{array}{c}58 \\
21.6 \%\end{array}$ & $\begin{array}{c}108 \\
40.1 \%\end{array}$ & $\begin{array}{c}73 \\
27.1 \%\end{array}$ & $\begin{array}{c}19 \\
7.1 \%\end{array}$ & $\begin{array}{l}11 \\
4.1 \%\end{array}$ & .240 & & \\
\hline $\begin{array}{l}\text { Entrepreneurial education } \\
\text { motivates students to start a } \\
\text { business }\end{array}$ & $\begin{array}{c}60 \\
22.3 \%\end{array}$ & $\begin{array}{c}113 \\
42.0 \%\end{array}$ & $\begin{array}{c}60 \\
22.3 \%\end{array}$ & $\begin{array}{c}22 \\
8.2 \%\end{array}$ & $\begin{array}{c}14 \\
5.2 \%\end{array}$ & .261 & & \\
\hline $\begin{array}{l}\text { I have gained lots of business } \\
\text { knowledge }\end{array}$ & $\begin{array}{c}47 \\
17.5 \%\end{array}$ & $\begin{array}{c}112 \\
41.6 \%\end{array}$ & $\begin{array}{c}85 \\
31.6 \%\end{array}$ & $\begin{array}{c}14 \\
5.2 \%\end{array}$ & $\begin{array}{l}11 \\
4.1 \%\end{array}$ & .239 & & \\
\hline
\end{tabular}

Null Hypotheses.4: There is no relationship between the choice of the respondents and Education.

From table No.6, it is observed that the p-value is less than 0.05 which means that the null hypothesis is rejected. This means that there is a clear relationship between the choices of the respondents and the Education. Comparing the K-S values that were obtained from the Kolmogorov-Smirnov test, it can be observed that 'Entrepreneurial education motivates students to start a business' ranked first (.261), followed by, 'Entrepreneurship education gives students business knowledge and better understanding' (.258), and 'Working in small business makes students gain real experience' (.252).

Table.7 Entrepreneurial intentions

\begin{tabular}{|c|c|c|c|c|c|c|c|c|}
\hline Statements & SA & $\mathbf{A}$ & $\mathbf{N}$ & D & SD & $\begin{array}{c}\mathrm{K}-\mathrm{S} \\
\text { value }\end{array}$ & $\chi^{2}$ & $\begin{array}{c}\text { p- } \\
\text { value }\end{array}$ \\
\hline $\begin{array}{l}\text { The graduates have the } \\
\text { intention to start a business }\end{array}$ & $\begin{array}{c}46 \\
17.1 \%\end{array}$ & $\begin{array}{c}105 \\
39.0 \%\end{array}$ & $\begin{array}{c}83 \\
39.9 \%\end{array}$ & $\begin{array}{c}20 \\
7.4 \%\end{array}$ & $\begin{array}{c}15 \\
5.6 \%\end{array}$ & .230 & \multirow{7}{*}{183.684} & \multirow{7}{*}{.000} \\
\hline $\begin{array}{l}\text { I would like to become a } \\
\text { successful businessman } \\
\text { /women in the future }\end{array}$ & $\begin{array}{c}100 \\
37.2 \%\end{array}$ & $\begin{array}{c}98 \\
36.4 \%\end{array}$ & $\begin{array}{c}48 \\
17.8 \%\end{array}$ & $\begin{array}{c}11 \\
4.1 \%\end{array}$ & $\begin{array}{c}12 \\
4.5 \%\end{array}$ & .244 & & \\
\hline $\begin{array}{l}\text { I intend to obtain a social } \\
\text { status as an entrepreneur }\end{array}$ & $\begin{array}{c}87 \\
32.3 \%\end{array}$ & $\begin{array}{c}91 \\
33.8 \%\end{array}$ & $\begin{array}{c}64 \\
23.8 \%\end{array}$ & $\begin{array}{c}15 \\
5.6 \%\end{array}$ & $\begin{array}{c}12 \\
4.5 \%\end{array}$ & .221 & & \\
\hline $\begin{array}{l}\text { I have a desire to start } \\
\text { entrepreneurial activities }\end{array}$ & $\begin{array}{c}69 \\
25.7 \% \\
\end{array}$ & $\begin{array}{c}103 \\
38.3 \%\end{array}$ & $\begin{array}{c}74 \\
27.5 \% \\
\end{array}$ & $\begin{array}{c}12 \\
4.5 \%\end{array}$ & $\begin{array}{c}11 \\
4.1 \%\end{array}$ & .229 & & \\
\hline $\begin{array}{l}\text { I would like to create my own } \\
\text { business }\end{array}$ & $\begin{array}{c}98 \\
36.4 \% \\
\end{array}$ & $\begin{array}{c}84 \\
31.2 \% \\
\end{array}$ & $\begin{array}{c}58 \\
21.6 \% \\
\end{array}$ & $\begin{array}{c}19 \\
7.1 \% \\
\end{array}$ & $\begin{array}{c}10 \\
3.7 \% \\
\end{array}$ & .215 & & \\
\hline $\begin{array}{l}\text { I would like to implement a } \\
\text { new business idea }\end{array}$ & $\begin{array}{c}76 \\
28.3 \% \\
\end{array}$ & $\begin{array}{c}104 \\
38.7 \% \\
\end{array}$ & $\begin{array}{c}62 \\
23.0 \% \\
\end{array}$ & $\begin{array}{c}13 \\
4.8 \% \\
\end{array}$ & $\begin{array}{c}14 \\
5.2 \% \\
\end{array}$ & .244 & & \\
\hline $\begin{array}{l}\text { I can successfully implement } \\
\text { the entrepreneurial process }\end{array}$ & $\begin{array}{c}64 \\
23.8 \%\end{array}$ & $\begin{array}{c}104 \\
38.7 \%\end{array}$ & $\begin{array}{c}59 \\
21.9 \%\end{array}$ & $\begin{array}{c}26 \\
9.7 \%\end{array}$ & $\begin{array}{c}16 \\
5.9 \%\end{array}$ & .248 & & \\
\hline
\end{tabular}


Null Hypotheses.5: There is no relationship between the choice of the respondents and the Entrepreneurial Intention.

From table No.7, it is observed that the p-value is less than 0.05 which means that the null hypothesis is rejected. This means that there is a clear relationship between the choices of the respondents and the Entrepreneurial Intention. Comparing the K-S values that were obtained from the Kolmogorov-Smirnov test, it can be observed that 'I can successfully implement the entrepreneurial process' ranked first (.248), followed by, 'I would like to implement a new business idea' \& 'I would like to become a successful businessman/women in the future' (.244), and 'The graduates have the intention to start a business' (.230).

Table 8. (a), (b), (c) and (d) Regression

Variables Entered/Removed ${ }^{\mathrm{a}}$

\begin{tabular}{|l|l|c|c|}
\hline Model & \multicolumn{1}{|c|}{ Variables Entered } & $\begin{array}{l}\text { Variables } \\
\text { Removed }\end{array}$ & Method \\
\hline 1 & $\begin{array}{l}\text { Education, Entrepreneurial } \\
\text { Potentials, Government Support, } \\
\text { Creativity }\end{array}$ & $\ldots$ & Enter \\
\hline
\end{tabular}

a Dependent variable: Entrepreneurial Intention

${ }^{b}$ All requested variables entered

Model Summary

\begin{tabular}{|c|c|c|c|c|}
\hline Model & R & R Square & $\begin{array}{c}\text { Adjusted R } \\
\text { Square }\end{array}$ & $\begin{array}{c}\text { Std. Error of } \\
\text { Estimate }\end{array}$ \\
\hline 1 & $.727^{\mathrm{a}}$ & .529 & .522 & 3.78306 \\
\hline
\end{tabular}

a Predictors: (Constant), Education, Entrepreneurial Potentials, Government Support, Creativity

ANOVA $^{\mathrm{a}}$

\begin{tabular}{|l|r|r|r|r|c|}
\hline & \multicolumn{1}{|c|}{$\begin{array}{c}\text { Sum of } \\
\text { Squares }\end{array}$} & \multicolumn{1}{c|}{ df } & \multicolumn{1}{c|}{$\begin{array}{c}\text { Mean } \\
\text { Square }\end{array}$} & \multicolumn{1}{c|}{ F } & Sig. \\
\hline Regression & 4178.185 & 4 & 1044.546 & 72.986 & $.000^{\mathrm{b}}$ \\
Residual & 3721.000 & 260 & 14.312 & & \\
Total & 7899.185 & 264 & & & \\
\hline
\end{tabular}

${ }^{\text {a }}$ Dependent variable: Entrepreneurial Intention

b Predictors: (Constant), Education, Entrepreneurial Potentials, Government Support, Creativity

\begin{tabular}{|l|r|r|c|r|r|}
\hline \multicolumn{1}{|c|}{ Coefficients $^{\mathbf{a}}$} \\
& \multicolumn{2}{|c|}{$\begin{array}{c}\text { Unstandardized } \\
\text { Coefficients }\end{array}$} & $\begin{array}{c}\text { Standardized } \\
\text { Coefficients }\end{array}$ & $\mathrm{t}$ & \multirow{2}{*}{ Sig. } \\
\cline { 2 - 6 } & \multicolumn{1}{|c|}{$\mathrm{B}$} & Std. Error & Beta & & \\
\hline (Constant) & 4.676 & 1.362 & & 3.433 & .001 \\
Entrepreneurial Potentials & .134 & .063 & .138 & 2.138 & .033 \\
Government Support Creativity & .088 & .076 & .077 & 1.154 & .249 \\
Education & .373 & .076 & .352 & 4.915 & .000 \\
& .240 & .075 & .242 & 3.218 & .001 \\
\hline
\end{tabular}

${ }^{a}$ Dependent variable: Entrepreneurial Intention

From the above co-efficient table, it can be seen that the p-value of Government support is > .05. Therefore, eliminating the variable government support, the linear regression analysis is done again and we get the results as follows: 
Table 9. (a), (b), (c) and (d) Regression

Variables Entered/Removed ${ }^{\text {a }}$

\begin{tabular}{|l|l|c|l|}
\hline Model & \multicolumn{1}{|c|}{ Variables Entered } & $\begin{array}{l}\text { Variables } \\
\text { Removed }\end{array}$ & Method \\
\hline 1 & $\begin{array}{l}\text { Education, Entrepreneurial } \\
\text { Potentials, Creativity }\end{array}$ & $\ldots$ & Enter \\
\hline
\end{tabular}

a Dependent variable: Entrepreneurial Intention

${ }^{b}$ All requested variables entered

\section{Model Summary}

\begin{tabular}{|c|c|c|c|c|}
\hline Model & $\mathbf{R}$ & R Square & $\begin{array}{c}\text { Adjusted R } \\
\text { Square }\end{array}$ & $\begin{array}{c}\text { Std. Error of } \\
\text { Estimate }\end{array}$ \\
\hline 1 & $.731^{\mathrm{a}}$ & .535 & .529 & 3.89009 \\
\hline
\end{tabular}

a Predictors: (Constant), Education, Entrepreneurial Potentials, Government Support, Creativity

\section{ANOVA ${ }^{\mathrm{a}}$}

\begin{tabular}{|l|r|r|r|r|l|}
\hline & \multicolumn{1}{|c|}{$\begin{array}{c}\text { Sum of } \\
\text { Squares }\end{array}$} & \multicolumn{1}{c|}{ df } & \multicolumn{1}{c|}{$\begin{array}{c}\text { Mean } \\
\text { Square }\end{array}$} & \multicolumn{1}{c|}{ F } & Sig. \\
\hline Regression & 4560.722 & 3 & 1523.541 & 100.678 & $.000^{\mathrm{b}}$ \\
Residual & 3979.918 & 263 & 15.133 & & \\
Total & 8550.540 & 266 & & & \\
\hline
\end{tabular}

${ }^{a}$ Dependent variable: Entrepreneurial Intention

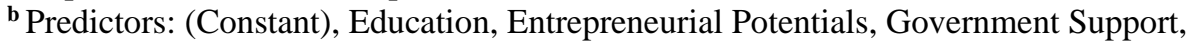
Creativity

\section{Coefficients $^{\mathrm{a}}$}

\begin{tabular}{|l|r|r|c|c|c|}
\hline & \multicolumn{2}{|c|}{$\begin{array}{c}\text { Unstandardized } \\
\text { Coefficients }\end{array}$} & $\begin{array}{c}\text { Standardized } \\
\text { Coefficients }\end{array}$ & \multirow{2}{*}{$\mathrm{t}$} & \multirow{2}{*}{ Sig. } \\
\cline { 2 - 5 } & \multicolumn{1}{|c|}{$\mathrm{B}$} & Std. Error & Beta & & \\
\hline (Constant) & 4.486 & 1.289 & & 3.481 & .001 \\
Entrepreneurial Potentials & .142 & .061 & .142 & 2.313 & .021 \\
Creativity & .439 & .075 & .406 & 5.819 & .000 \\
Education & .256 & .071 & .253 & 3.620 & .000 \\
\hline
\end{tabular}

${ }^{a}$ Dependent variable: Entrepreneurial Intention

The $\mathrm{R}^{2}$ value of .535 shows that $53.5 \%$ of the samples are influenced by the linear regression. From F-table, it is clear that the p-value is $.000<.05$ which confirms that there is a linear relationship between the dependent and independent variables. Therefore, the obtained linear regression is as follows:

$\mathrm{EI}=4.486+.142 \mathrm{EP}+.439 \mathrm{C}+.256 \mathrm{E}$

Where EI is the Entrepreneurial Intention, EP is the Entrepreneurial Potentials, $\mathrm{C}$ is the Creativity and E is the Education.

Thus, it can be seen that the Entrepreneurial intention is influenced by the Graduate Potentials, Creativity, and Education of the students and not by the Government support. i.e. the claimed hypothesis is proved.

\section{Discussion}


Most of the respondents $(72.1 \%)$ wants to establish new businesses. $64.3 \%$ of them responded that they are confident in starting their own businesses. The main reason for their intention to start the new businesses was the training and development opportunities (26\%), followed by their expectation of financial support (19.7\%) and expected friends support $(19.3 \%)$ in establishing new businesses.

Among the graduate potentials, it was found that most of the graduates have the confidence to start a business and they also believed that the graduates have the skills to take responsibility, and the ability to accomplish several tasks.

Among the Government support factors, it was found that most of the students believed that new ideas of the students need Government support to put into use and emotional support from family and friends makes them feel proud.

Among the creativity factors, most of them believed that the students can find solutions to problems and have the ability to present ideas, and can do business in a new innovative way.

Among the educational factors, the majority of the students believed that entrepreneurship education motivates the students to start a business as it gives knowledge and a better understanding of business, and working in small businesses makes the students gain real-life experience.

Among the entrepreneurial intention factors, most of the students believed that they can successfully implement the entrepreneurial process as they would like to implement a new business idea and they would like to become successful businessmen/women in the future.

It was proved that Graduate Potentials, Creativity, and Education impacts the Entrepreneurial Intention of the students but not the Government support i.e. Entrepreneurial intention is influenced by Graduate Potentials, Creativity, and Education of the students.

\section{Conclusion}

Entrepreneurship is an increasingly popular feature of modern society today. Entrepreneurship seeds in students the qualities like creativity, self-confidence, ambition, and the ability to take risks. The study confirms that undergraduate students are developing creative skills and bringing out their entrepreneurial potentials and improvising their practical knowledge through their education which is in line with the findings by Khan (2008). These qualities help to develop society and keep pace with the development and technological changes in the environment through entrepreneurial intention among the students. Indeed, the motivation for the student to achieve their dreams comes up with their ideas taught by the teachers educating courses like entrepreneurship education and the expected support from the educational authorities and government which is similar to the findings of Al Buraiki and Khan (2018). They create opportunities to satisfy the needs of individuals through establishing new markets and are creative in promoting innovative methods.

\section{Suggestions}

The current study is concerned with entrepreneurship for students who are about to graduate. Hence, the following suggestions are made:

1. The main focus of the graduating students should be towards creativity encompassing innovation and practical usage so that the realm of entrepreneurship emerges out.

2. The graduating students developing creative skills and enhancing their entrepreneurial potential through their collegiate education whereas they need proper guidance to fulfill their entrepreneurial intention which is in the hands of teachers.

3. Students should be exposed to the development of various skills - creativity, communication, presentation, marketing, funds management, etc.

4. Graduating students should be invited to a platform where competitions of all innovative kinds should be conducted regularly and the participants should be guided with assistance to improve their ideas.

5. Business knowledge with more focus on practical experience should be initiated rather than sharing simply theoretical knowledge.

6. The government should encourage students through various schemes so that the graduate students will come forward to take up new ventures with their innovative ideas.

\section{References}

1. Al Buraiki, A., \& Khan, F. R. (2018). Finance and Technology: Key Challenges faced by Small and Medium Enterprises (SMEs) in Oman. International Journal of Management, Innovation \& Entrepreneurial Research, 4(2), 1-12. http://doi.org/10.18510/ijmier.2018.421

2. Ács, Z., Szerb, L., \& Autio, E. (2014). Global Entrepreneurship and Development Index 2014 Report| Global Entrepreneurship Development Institute. [online] Thegedi. org. 
3. Al-Shanfari, D. A. (2012, January). Entrepreneurship in Oman: A snapshot of the main challenges. In Proceedings of the United Nations conference on trade and development: Multi-year expert meeting on enterprise development policies and capacity-building in science, technology, and innovation (STI)(fourth session), Geneva, Switzerland: Geneva.

4. Audretsch, D. B., \& Thurik, A. R. (2001). What's new about the new economy? Sources of growth in the managed and entrepreneurial economies. Industrial and corporate change, 10(1), 267-315.

5. Bindah, E. V. \& Magd, H. A. (2016). Teaching entrepreneurship in Oman: successful approaches. Procedia-Social and Behavioral Sciences, 219, 140-144.

6. Bosma, N., Sanders, M., \& Stam, E. (2018). Institutions, entrepreneurship, and economic growth in Europe. Small Business Economics, 51(2), 483-499.

7. Cowling, M., \& Taylor, M. (2001). Entrepreneurial women and men: two different species?. Small Business Economics, 16(3), 167-175.

8. Delmar, F., \& Davidsson, P. (2000). Where do they come from? Prevalence and characteristics of nascent entrepreneurs. Entrepreneurship \& regional development, 12(1), 1-23.

9. Durrah, O. M., Allil, K. K., \& Alkhalaf, T. (2018). The intellectual capital and the learning organization. International Journal of Public Leadership., 14(2), 109-118.

10. El Talla, S. A., Al Shobaki, M. J., Abu-Naser, S. S., \& Amuna, Y. M. A. (2018). Crowdfunding Role in Boosting the Entrepreneurial Creativity of University Students, 2(4), 1-12.

11. Ennis, C. A. (2015). Between trend and necessity: Top-down entrepreneurship promotion in Oman and Qatar. The Muslim World, 105(1), 116-138.

12. Fayolle, A., Verzat, C., \& Wapshott, R. (2016). In quest of legitimacy: The theoretical and methodological foundations of entrepreneurship education research. International Small Business Journal, 34(7), 895-904.

13. Galloway, L., \& Brown, W. (2002). Entrepreneurship education at university: a driver in the creation of high growth firms? Education+ Training, 44(8/9), 398-405. https://doi.org/10.1108/00400910210449231

14. Galloway, L., Anderson, M., \& Brown, W. (2006). Are engineers becoming more enterprising? A study of the potentials of entrepreneurship education. International Journal of Continuing Engineering Education and Life-Long Learning, 16(5), 355-365.

15. Gurol, Y., \& Atsan, N. (2006). Entrepreneurial Characteristics Amongst University Student: Some Insights For Entrepreneurship Education And Training In Turkey, Education and Training, 48(1), $1-22$.

16. Haddad, M. I., Williams, I. A., Hammoud, M. S., \& Dwyer, R. J. (2019). Strategies for implementing innovation in small and medium-sized enterprises. World Journal of Entrepreneurship, Management, and Sustainable Development, 16(1), 12-29. https://doi.org/10.1108/WJEMSD-05-2019-0032

17. Hamdan, A. M. M. (2019). Entrepreneurship and Economic Growth: An Emirati Perspective. The Journal of Developing Areas, 53(1). http://doi.org/10.1353/jda.2019.0004

18. Hunjra, A. I., Ahmad, H. M., \& Niazi, G. S. K. (2011). Planned behavior entrepreneurship and intention to create a new venture among young graduates. Kashif ur, Planned Behavior Entrepreneurship, and Intention to Create New Venture Among Young Graduates (September 23, 2011).

19. Ibrahim, O. A., Devesh, S., \& Ubaidullah, V. (2017). Implication of attitude of graduate students in Oman towards entrepreneurship: an empirical study. Journal of Global Entrepreneurship Research, 7(1), 8. https://doi.org/10.1186/s40497-017-0066-2

20. Khan, F.R. (2008). The effectiveness of Entrepreneurship Knowledge and Training: An empirical study in relates to Chennai (South India). Proceedings of the AGBA Fifth World Congress, Bahrain, ISSN 1549-9332, 5 (1) Part. 2, 483-502, available at http://www.agba.us/pdf/AGBA-2008-Bahrainvol.2-proceedings.pdf

21. Khan, F. R. (2014). Socio-economic factors influencing entrepreneurship development: An empirical study across the small and medium enterprises of Chennai, State of Tamil Nadu, India. International Journal of Students Research in Technology \& Management, 2(03), 89-94.

22. Khan, G. M., \& Almoharby, D. (2007). Towards enhancing entrepreneurship development in Oman. Journal of enterprising culture, 15(04), 371-392.

23. Khan, S. A., Ghosh, A. P., \& Myers, D. A. (2005, June). Women entrepreneurship in Oman. In Proceedings of 50th World Conference of the International Council for the Small Business (ICSB), 15-18.

24. Krueger Jr, N. F., \& Brazeal, D. V. (1994). Entrepreneurial potential and potential entrepreneurs. Entrepreneurship theory and practice, 18(3), 91-104. 
25. Lüthje, C., \& Franke, N. (2003). The 'making' of an entrepreneur: testing a model of entrepreneurial intent among engineering students at MIT. R\&d Management, 33(2), 135-147.

26. Mathisen, G. E., Einarsen, S., \& Mykletun, R. (2012). Creative leaders promote creative organizations. International Journal of Manpower, 33(4), 367-382.

27. Matlay, H., Belwal, R., Al Balushi, H., \& Belwal, S. (2015). Students' perception of entrepreneurship and enterprise education in Oman. Education+ Training, 57(8/9), 924-947, https://doi.org/10.1108/ET-12-2014-0149

28. Marques, A. P., \& Moreira, R. (2013). 'Traditional' and 'Innovative' Potential Entrepreneurs among Portuguese Graduates: A Case Study. Journal of Educational and Social Research, 3(7), 134.

29. Matriano, M. T., \& Suguku, D. (2015). Entrepreneurship Growth in Oman: Position, Prospects, and Growth of Entrepreneurial Education. International E-Journal of Advances in Education, 1(2), 127 131.

30. Postigo, S., Iacobucci, D., \& Tamborini, M. F. (2003, September). Undergraduate students as a source of potential entrepreneurs: a comparative study between Italy and Argentina. In 13th global IntEnt-internationalizing entrepreneurship education and training conference (pp. 8-10).

31. Sahay, A., \& Rai, S. K. (2004). How entrepreneurial are youth of Varanasi: An empirical study through cyber cafes. Journal of Services Research, 4(2), 175-203.

32. Santarelli, E., \& Vivarelli, M. (2007). Entrepreneurship and the process of firms' entry, survival, and growth. Industrial and corporate change, 16(3), 455-488.

33. Sokol, A., Gozdek, A., Figurska, I., \& Blaskova, M. (2015). Organizational climate of higher education institutions and its implications for the development of creativity. Procedia-Social and Behavioral Sciences, 182, 279-288.

34. Valeri, M. (2013). Oligarchy vs. Oligarchy: Business and politics of reform in Bahrain and Oman. Business politics in the Middle East, 17-42.

35. Varghese, T., \& Hassan, A. (2012). Youth's entrepreneurial attitudes in Oman. World Journal of Social Sciences, 2(7), 302-325.

36. Wong, W. K., Cheung, H. M., \& Venuvinod, P. K. (2005). Individual entrepreneurial characteristics and entrepreneurial success potential. International Journal of Innovation and Technology Management, 2(03), 277-292. https://doi.org/10.1142/S0219877005000502

37. Yarahmadi, F., \& Magd, H. A. (2016). Entrepreneurship infrastructure and education in Oman. Procedia-Social and Behavioral Sciences, 219, 792-797.

38. Y1lmaz, E. (2010). The analysis of organizational creativity in schools regarding principals' ethical leadership characteristics. Procedia-Social and Behavioral Sciences, 2(2), 3949-3953.

39. Zeffane, R. (2013). Gender and youth entrepreneurial potential: Evidence from the United Arab Emirates. International Journal of Business and Management, 8(1), 60.

Copyright: () 2020 by the authors. Licensee Global Scientific Publications, Oman.

This work is licensed under a Creative Commons Attribution-ShareAlike 4.0 International License.

This is an open-access journal and the articles published in this journal are distributed under the terms of CC-BY-SA. 